\title{
Border Figure Detection Using a Phase Oscillator Network with Dynamical Coupling
}

\author{
L. H. A. Monteiro, ${ }^{1,2}$ I. Gonzalez, ${ }^{1}$ and J. R. C. Piqueira ${ }^{2}$ \\ ${ }^{1}$ Pósgraduação em Engenharia Elétrica, Escola de Engenharia, Universidade Presbiteriana Mackenzie, \\ Rua da Consolação 896, CEP 01302-907, São Paulo, SP, Brazil \\ ${ }^{2}$ Departamento de Engenharia de Telecomunicações e Controle, Escola Politécnica, Universidade de São \\ Paulo, Av. Prof. Luciano Gualberto 380, Travessa 3, CEP 05508-900, São Paulo, SP, Brazil
}

Correspondence should be addressed to L. H. A. Monteiro, luizm@mackenzie.br

Received 13 January 2008; Revised 17 April 2008; Accepted 30 May 2008

Recommended by Jerzy Warminski

\begin{abstract}
Oscillator networks have been developed in order to perform specific tasks related to image processing. Here we analytically investigate the existence of synchronism in a pair of phase oscillators that are short-range dynamically coupled. Then, we use these analytical results to design a network able of detecting border of black-and-white figures. Each unit composing this network is a pair of such phase oscillators and is assigned to a pixel in the image. The couplings among the units forming the network are also dynamical. Border detection emerges from the network activity.

Copyright $@ 2008$ L. H. A. Monteiro et al. This is an open access article distributed under the Creative Commons Attribution License, which permits unrestricted use, distribution, and reproduction in any medium, provided the original work is properly cited.
\end{abstract}

\section{Introduction}

The synchronous firing of neurons seems to be important for accomplishing cognitive tasks such as attention (e.g., [1]), comprehension (e.g., [2]), coordination (e.g., [3]), perception (e.g., [4]), and sensory segmentation (e.g., [5]). Such experimental findings have inspired works about image processing (e.g., recognition, segmentation, symmetry detection) based on the synchronism of coupled oscillators (e.g., [6-11]). In addition, several neural systems, such as spinal cord (e.g., $[12,13])$, hippocampus (e.g., $[14,15])$, and visual cortex (e.g., $[16,17])$, have been modelled by phase oscillators-one of the simplest oscillator models. In spite of its simplicity, this approach is suitable because more complex models for neurons like pulsecoupled and Hodgkin-Huxley-type models can be transformed into a phase oscillator model through coordinate changes [18]. Networks of phase oscillators can be electronically built using phase-locked loops (PLLs) (e.g., [19-22]).

Here we analytically study a phase oscillator model with dynamical coupling, and then use it in order to form a network capable of successfully detecting border of black-and-white 
figures. Each oscillator of our network corresponds to a first-order PLL (e.g., [8, 11, 17, 23]), which is equivalent to an overdamped pendulum.

The aim of any border detection process is to capture the main structural properties of an image, which can be useful, for instance, for image compression or diagnosis in echocardiography (e.g., [24]). Usually, this kind of image processing involves the use of partial derivatives (gradient, Laplacian; e.g., [25]), which are discretized in the space domain and in the time domain. Here we propose a scheme based on a network where each unit is formed by a pair of dynamically coupled PLLs corresponding to a pixel of the image. Border detection is obtained from the network activity. Notice that such a model can be transformed into a dedicated hardware for executing this image processing task. Also it naturally presents space discretization, because it is composed of a finite number of PLLs. This feature plus the fact that the time domain does need to be discretized can reduce the stability problems related to the algorithms employed for numerically calculating partial derivatives.

\section{Model of a single unit}

Consider that the temporal activities of two first-order PLLs with dynamical coupling are described by the following equations:

$$
\begin{aligned}
\frac{d \theta_{1}}{d t} & =\omega_{1}+k_{12} \sin \left(\theta_{2}-\theta_{1}\right), \\
\frac{d \theta_{2}}{d t} & =\omega_{2}+k_{21} \sin \left(\theta_{1}-\theta_{2}\right), \\
\frac{d k_{12}}{d t} & =\mu \alpha_{1} \cos \left(\theta_{1}-\theta_{2}\right)-\mu k_{12}, \\
\frac{d k_{21}}{d t} & =\mu \alpha_{2} \cos \left(\theta_{2}-\theta_{1}\right)-\mu k_{21},
\end{aligned}
$$

where $\theta_{j}$ and $\omega_{j}(j=1,2)$ are the phase and the natural frequency of the oscillator $j$, respectively; $k_{i j}(i, j=1,2$ with $i \neq j)$ is the connection strength from the oscillator $j$ to the oscillator $i ; \alpha_{j}(j=1,2)$ is the coefficient related to the Hebbian connection modification; and $\mu$ represents a natural (exponential) decay. The parameters $\omega_{j}, \alpha_{j}$, and $\mu$ are positive numbers. Thus, the connection strengths are enhanced when the oscillators are in phase, and they are weakened when they are out of phase. This kind of (synaptic) modification between two oscillators (neurons) was suggested by Hebb [26].

By defining $q \equiv \theta_{2}-\theta_{1} ; \omega \equiv \omega_{2}-\omega_{1} ; \alpha_{0} \equiv\left(\alpha_{1}+\alpha_{2}\right) / 2 ; k \equiv\left(k_{12}+k_{21}\right) / 2$, system $(2.1)$ can be rewritten as

$$
\begin{aligned}
& \frac{d q}{d t}=f_{1}(q, k)=\omega-2 k \sin q, \\
& \frac{d k}{d t}=f_{2}(q, k)=\mu \alpha_{0} \cos q-\mu k .
\end{aligned}
$$

The variables $q$ and $k$ represent the phase difference and the average connection strength, respectively. Notice that $f_{j}(q, k)=f_{j}(q+2 \pi, k)(j=1,2)$. The formation of synchronized clusters in networks of phase oscillators described by system (2.2) was investigated by Seliger et al. [27]. In this section we study the asymptotic behaviors of this model. 
Synchronism occurs when both nodes oscillate in a common frequency, which means that $d \theta_{2} / d t=d \theta_{1} / d t$ or $\theta_{2}-\theta_{1}=q=$ constant. Thus, synchronism can happen if there are constants $\left(q^{*}, k^{*}\right)$ so that if $(q(t), k(t))=\left(q^{*}, k^{*}\right)$ then $d q / d t=0$ and $d k / d t=0$ for all time $t$. Notice that a synchronous solution is an equilibrium point $\left(q^{*}, k^{*}\right)$ of the dynamical system (2.2). Such a solution corresponds to an intersection point of the nullclines $f_{1}=0$ $(k=\omega /(2 \sin q))$ and $f_{2}=0\left(k=\alpha_{0} \cos q\right)$.

The stability of an equilibrium point $\left(q^{*}, k^{*}\right)$ can usually be determined by calculating the eigenvalues $\lambda$ of the Jacobian matrix corresponding to system (2.2) linearized around this point. It is asymptotically stable when all eigenvalues have negative real parts (e.g., [28]). For system (2.2), the eigenvalues $\lambda$ are the roots of the polynomial

$$
\lambda^{2}+\left(\mu+2 k^{*} \cos q^{*}\right) \lambda+2 \mu k^{*} \cos q^{*}-2 \mu \alpha_{0}\left(\sin q^{*}\right)^{2}=0 .
$$

According to the Routh-Hurwitz criterion, both roots of the polynomial $\lambda^{2}+a_{1} \lambda+a_{2}=0$ have negative real parts if $a_{1}>0$ and $a_{2}>0$ (e.g., [29]).

When $\omega=0$, the equilibrium points are $P_{1}=\left(0, \alpha_{0}\right), P_{2}=(\pi / 2,0), P_{3}=\left(\pi,-\alpha_{0}\right), P_{4}=$ $(3 \pi / 2,0)$, where $P_{1}$ and $P_{3}$ are asymptotically stable, and $P_{2}$ and $P_{4}$ are unstable.

When $\omega \neq 0$, there are also four equilibrium solutions if $\omega<\alpha_{0}$. The points with

$$
k^{*}= \pm \frac{\alpha_{0}}{\sqrt{2}} \sqrt{1+\sqrt{1-\left(\frac{\omega}{\alpha_{0}}\right)^{2}}}
$$

are asymptotically stable; the ones with

$$
k^{*}= \pm \frac{\alpha_{0}}{\sqrt{2}} \sqrt{1-\sqrt{1-\left(\frac{\omega}{\alpha_{0}}\right)^{2}}}
$$

are unstable. When $\omega=\alpha_{0}$, there are only two equilibrium points because two saddle-node bifurcations (e.g., [28]) occur. When $\omega>\alpha_{0}$, there is not synchronism; however, there is a limit cycle, which corresponds to a closed and isolated trajectory in the state space $q \times k$.

For an autonomous two-dimensional system, Poincaré-Bendixson theorem (e.g., [28]) ensures that there is an asymptotically stable limit cycle in a region of the state space if the vector field $\left(f_{1}, f_{2}\right)$ points inward everywhere on the boundary of this region, which must not contain any equilibrium point. For system (2.2), it is easy to verify that if $\omega>\alpha_{0}$, then there is such an attracting trajectory inside the region $R$, where $R$ is the rectangle given by $R=\left\{(q, k): 0 \leq q<2 \pi, \alpha_{0}+(\epsilon / \mu)<k<-\alpha_{0}-(\epsilon / \mu)\right\}$ for $\epsilon \rightarrow 0_{+}$. In this case, $k$ oscillates and $q$ grows as the time goes by, as shown in Figure 2. Figure 1 illustrates the case with synchronous (equilibrium) asymptotic solution.

In order to analytically characterize the limit cycle, suppose that the asymptotic behavior of $q(t)$ in this case is given by $q_{\text {asymp }}(t) \simeq B t$. By inserting this approximated (linear) solution in the equation for $d k / d t$ and integrating it, the asymptotic solution of $k(t)$ is

$$
k_{\text {asymp }}(t) \simeq A \cos (B t-\varphi),
$$

where $A=\mu \alpha_{0} / \sqrt{\mu^{2}+B^{2}}$ and $\sin \varphi=B / \sqrt{\mu^{2}+B^{2}}$. By substituting this expression in the equation for $d q / d t$ in (2.2), an equation for calculating $B$ is found. In fact, $B$ is the root of the polynomial

$$
B^{3}-\omega B^{2}+\left(\mu^{2}+\mu \alpha_{0}\right) B-\omega \mu^{2}=0
$$



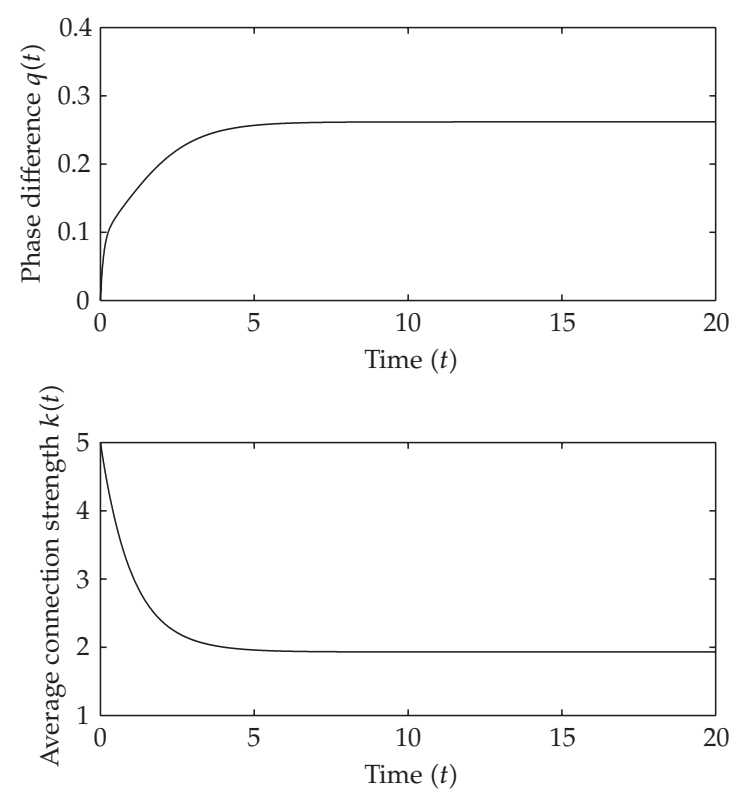

Figure 1: Temporal evolution of $q(t)$ and $k(t)$ obtained by numerically integrating system (2.2). Parameter values: $\omega=1, \alpha=2, \mu=1$. Initial conditions: $q(0)=0, k(0)=5$. In this case, the asymptotic solution corresponds to the equilibrium point $\left(q^{*}, k^{*}\right)=(0.26,1.93)$.
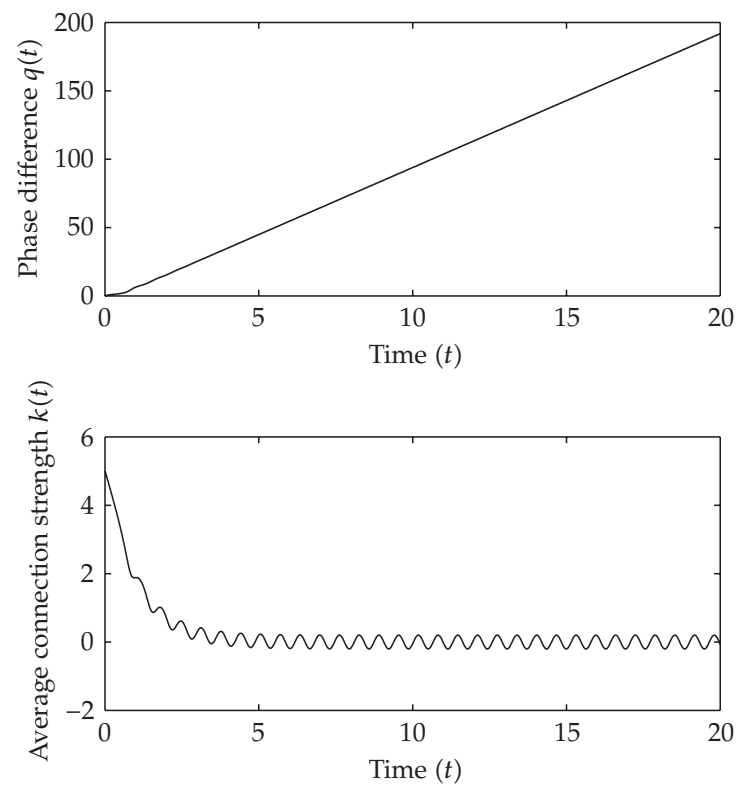

Figure 2: Temporal evolution of $q(t)$ and $k(t)$ obtained by numerically integrating system (2.2). Parameter values: $\omega=10, \alpha=2, \mu=1$. Initial conditions: $q(0)=0, k(0)=5$. In this case, the asymptotic solution corresponds to the limit cycle, where $k(t)$ oscillates with frequency $B \simeq 9.8$ and amplitude $A \simeq 0.20$, and $q(t)$ linearly grows with slope $A$. 


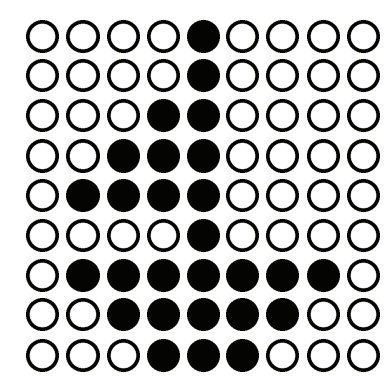

(a)

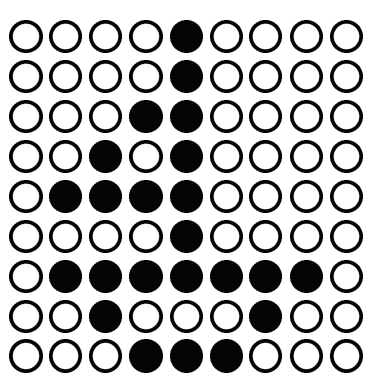

(b)

Figure 3: (a) At $t=0$, this is the image presented to the network with $9 \times 9$ units. (b) At $t=40$, the border of the image is dynamically determined.

Observe that when $\omega \gg 1$, then the angular velocity $B$ can be written as $B \simeq \omega+\Delta$, with $|\Delta| \ll 1$. The value of $\Delta$ is estimated by

$$
\Delta \simeq-\frac{\mu \alpha_{0} \omega}{\omega^{2}+\mu^{2}+\mu \alpha_{0}}
$$

Consequently, the oscillation amplitude $A$ of $k_{\text {asymp }}(t)$ when $\omega \gg 1$ is

$$
A \simeq \frac{\mu \alpha_{0}}{\omega}
$$

For instance, for $\omega=10, \alpha_{0}=2$, and $\mu=1$, these approximated expressions give $B \simeq 9.8$ and $A \simeq 0.20$, which are in good agreement with the numerical solutions of system (2.2) shown in Figure 2.

\section{Network for detecting figure border}

Our two-dimensional network for detecting figure border is composed of units consisting of a pair of phase oscillators forced by an external input $I$. In this network, the coefficients $\alpha_{1}$ and $\alpha_{2}$ can vary with the time. Thus, system (2.2) is rewritten as

$$
\begin{aligned}
& \frac{d q}{d t}=I+\omega-2 k \sin q, \\
& \frac{d k}{d t}=\mu \alpha(t) \cos q-\mu k .
\end{aligned}
$$

Assume that a black-and-white figure will be presented to this network, as illustrated in Figure 3(a). The variable $I$ represents the input for each unit according to the following rules: $I=0$ corresponds to a white part of the figure; $I=9$ corresponds to a black part. Notice that the input can be translated into a new natural frequency $\Omega$ by defining $I+\omega \equiv \Omega$. Thus, system (3.1) is reduced to system (2.2) if $\alpha(t)$ is a constant.

Each unit is coupled with its four closest neighbors (left, right, up, and down), which is usually known as four-neighborhood in image processing literature (e.g., [30]) or von 
Neumann neighborhood in cellular automaton literature (e.g., [31]). As a consequence of this coupling, the value of $\alpha(t)$ for each cell is given by

$$
\alpha(t)= \begin{cases}\alpha_{0} & \text { if } 0 \leq t \leq T \\ p \alpha_{0} & \text { if } t>T\end{cases}
$$

The parameter $T$ is a settling time; $p \equiv 1+q x$ where $q$ is a positive number (here $q=14$ ); $x=G(y)$ where $G(y)=0$ if $y=0$; and $G(y)=1$ if $y \neq 0$. The value of $y$ is obtained by

$$
y=\prod_{i=1}^{4}\left[k_{i}(T)-k_{i}(T-\delta T)\right],
$$

where $\delta T$ is the time step (here $\delta T=0.01$ ) of the integration method (here fourth-order Runge-Kutta method) used for numerically solving the dynamical system. The variable $k_{i}(t)$ corresponds to the average connection strength of the closest neighbor $i(i=1,2,3,4)$. If the neighbor $j$ does not exist, then $k_{j}(t) \equiv 0$.

An image is presented to the network at $t=0$. For $0 \leq t \leq T$, all the units behave as if they were isolated, because the value of $\alpha\left(=\alpha_{0}\right)$ for each unit is independent of the neighbor activity. By taking for all units $\omega=1, \alpha_{0}=2, \mu=1, T=20, q(0)=0$, and $k(0)=5$, the units with $I=0$ will tend to a stationary solution and the units with $I=9$ will tend to a limit cycle, as explained in the last section. For these parameter values, the permanent regime was already reached when $t=T$, as shown in Figures 1 and 2.

At $t=T$, the value of $\alpha$ for each unit can be changed. Expressions (3.2) and (3.3) imply what follows. If all of its four neighbors are in a limit cycle $(y \neq 0)$, then the value of $\alpha$ is increased from 2 to 30; if at least one neighbor is in a stationary state or if the unit does not have a complete von Neumann neighborhood ( $y=0$ ), then the value of $\alpha$ is kept equal to 2 . The limit cycle is characterized by $k(t)$ oscillating with frequency $B$ and amplitude $A$, and $q(t)$ linearly growing with slope $A$, as presented in the last section.

For $\alpha=30$ and $\Omega=10, q(t)$ and $k(t)$ tend to a stationary activity, which in our model corresponds to the white color. Thus, at $t=2 T=40$, the unique units remaining in oscillatory activity (the black color) are the ones in the figure border. Figure 3(b) shows the contour of Figure 3(a), after the units have reached the (new) permanent regime.

The higher the value of $\mu$ is, the shorter the transitory phase will be. Hence, border detection can be made faster by increasing the value of this parameter.

\section{Conclusion}

Networks governed by differential (e.g., $[6-8,10,11])$ or difference equations (e.g., $[9,32])$ have been employed for image processing. Here we used a network with local and dynamical coupling for identifying contour of black-and-white images. The unit of such a network is the variant of the Kuramoto model [33] for two oscillators (neurons), which was proposed by Seliger et al. [27]. In our scheme, after applying the input corresponding to the figure, the value of $\alpha$ for each unit is switched or not, depending on the neighborhood activity. This scheme could be implemented using first-order PLLs. This investigation could be extended for higherorder PLLs, which could be designed in order to shorten the transient. 


\section{Acknowledgment}

L. Monteiro and J. Piqueira are partially supported by CNPq.

\section{References}

[1] P. N. Steinmetz, A. Roy, P. J. Fitzgerald, S. S. Hsiao, K. O. Johnson, and E. Niebur, "Attention modulates synchronized neuronal firing in primate somatosensory cortex," Nature, vol. 404, no. 6774, pp. 187190, 2000.

[2] M. A. Just, V. L. Cherkassky, T. A. Keller, and N. J. Minshew, “Cortical activation and synchronization during sentence comprehension in high-functioning autism: evidence of underconnectivity," Brain, vol. 127, no. 8, pp. 1811-1821, 2004.

[3] S. N. Baker, J. M. Kilner, E. M. Pinches, and R. N. Lemon, "The role of synchrony and oscillations in the motor output," Experimental Brain Research, vol. 128, no. 1-2, pp. 109-117, 1999.

[4] E. Rodriguez, N. George, J.-P. Lachaux, J. Martinerie, B. Renault, and F. J. Varela, "Perception's shadow: long-distance synchronization of human brain activity," Nature, vol. 397, no. 6718, pp. 430433, 1999.

[5] A. K. Engel, P. König, A. K. Kreiter, T. B. Schillen, and W. Singer, "Temporal coding in the visual cortex: new vistas on integration in the nervous system," Trends in Neurosciences, vol. 15, no. 6, pp. 218-226, 1992.

[6] Y. Hayashi, "Oscillatory neural network and learning of continuously transformed patterns," Neural Networks, vol. 7, no. 2, pp. 219-231, 1994.

[7] D. L. Wang, "Emergent synchrony in locally coupled neural oscillators," IEEE Transactions on Neural Networks, vol. 6, no. 4, pp. 941-948, 1995.

[8] F. C. Hoppensteadt and E. M. Izhikevich, "Pattern recognition via synchronization in phase-locked loop neural networks," IEEE Transactions on Neural Networks, vol. 11, no. 3, pp. 734-738, 2000.

[9] R. de Oliveira and L. H. A. Monteiro, "Symmetry detection using global-locally coupled maps," in Proceedings of the 12th International Conference on Artificial Neural Networks (ICANN '02), vol. 2415 of Lecture Notes in Computer Science, pp. 75-80, Madrid, Spain, August 2002.

[10] M. Ursino, G.-E. La Cara, and A. Sarti, "Binding and segmentation of multiple objects through neural oscillators inhibited by contour information," Biological Cybernetics, vol. 89, no. 1, pp. 56-70, 2003.

[11] B. K. Ghosh, A. D. Polpitiya, and W. Wang, "Bio-inspired networks of visual sensors, neurons, and oscillators," Proceedings of the IEEE, vol. 95, no. 1, pp. 188-214, 2007.

[12] A. H. Cohen, P. J. Holmes, and R. H. Rand, "The nature of the coupling between segmental oscillators of the lamprey spinal generator for locomotion: a mathematical model," Journal of Mathematical Biology, vol. 13, no. 3, pp. 345-369, 1981.

[13] D. Taylor and P. Holmes, "Simple models for excitable and oscillatory neural networks," Journal of Mathematical Biology, vol. 37, no. 5, pp. 419-446, 1998.

[14] R. M. Borisyuk and F. C. Hoppensteadt, "Memorizing and recalling spatial-temporal patterns in an oscillator model of the hippocampus," BioSystems, vol. 48, no. 1-3, pp. 3-10, 1998.

[15] F. K. Skinner, C. Wu, and L. Zhang, "Phase-coupled oscillator models can predict hippocampal inhibitory synaptic connections," European Journal of Neuroscience, vol. 13, no. 12, pp. 2183-2194, 2001.

[16] Y. B. Kazanovich and R. M. Borisyuk, "Dynamics of neural networks with a central element," Neural Networks, vol. 12, no. 3, pp. 441-454, 1999.

[17] L. H. A. Monteiro, N. C. F. Canto, J. G. Chaui-Berlinck, F. M. Orsatti, and J. R. C. Piqueira, "Global and partial synchronism in phase-locked loop networks," IEEE Transactions on Neural Networks, vol. 14, no. 6, pp. 1572-1575, 2003.

[18] E. M. Izhikevich, "Weakly pulse-coupled oscillators, FM interactions, synchronization, and oscillatory associative memory," IEEE Transactions on Neural Networks, vol. 10, no. 3, pp. 508-526, 1999.

[19] J. R. C. Piqueira, F. M. Orsatti, and L. H. A. Monteiro, "Computing with phase locked loops: choosing gains and delays," IEEE Transactions on Neural Networks, vol. 14, no. 1, pp. 243-247, 2003.

[20] L. H. A. Monteiro, D. N. F. Filho, and J. R. C. Piqueira, "Bifurcation analysis for third-order phaselocked loops," IEEE Signal Processing Letters, vol. 11, no. 5, pp. 494-496, 2004.

[21] J. R. C. Piqueira, M. Q. Oliveira, and L. H. A. Monteiro, "Synchronous state in a fully connected phaselocked loop network," Mathematical Problems in Engineering, vol. 2006, Article ID 52356, 12 pages, 2006. 
[22] J. R. C. Piqueira and M. D. C. Freschi, "Models for master-slave clock distribution networks with thirdorder phase-locked loops," Mathematical Problems in Engineering, vol. 2007, Article ID 18609, 17 pages, 2007.

[23] L. H. A. Monteiro, R. V. dos Santos, and J. R. C. Piqueira, "Estimating the critical number of slave nodes in a single-chain PLL network," IEEE Communications Letters, vol. 7, no. 9, pp. 449-450, 2003.

[24] J. N. Kirkpatrick, R. M. Lang, S. E. Fedson, A. S. Anderson, J. Bednarz, and K. T. Spencer, "Automated border detection on contrast enhanced echocardiographic images," International Journal of Cardiology, vol. 103, no. 2, pp. 164-167, 2005.

[25] G. Aubert and P. Kornprobst, Mathematical Problems in Image Processing, vol. 147 of Applied Mathematical Sciences, Springer, New York, NY, USA, 2nd edition, 2006.

[26] D. O. Hebb, The Organization of Behavior, John Wiley \& Sons, New York, NY, USA, 1949.

[27] P. Seliger, S. C. Young, and L. S. Tsimring, "Plasticity and learning in a network of coupled phase oscillators," Physical Review E, vol. 65, no. 4, Article ID 041906, 7 pages, 2002.

[28] J. Guckenheimer and P. Holmes, Nonlinear Oscillations, Dynamical Systems, and Bifurcations of Vector Fields, vol. 42 of Applied Mathematical Sciences, Springer, New York, NY, USA, 1983.

[29] K. Ogata, Modern Control Engineering, Prentice-Hall, Upper Saddle River, NJ, USA, 2001.

[30] R. C. Gonzalez and R. E. Woods, Digital Image Processing, Addison-Wesley, New York, NY, USA, 2007.

[31] S. Wolfram, Ed., Theory and Applications of Cellular Automata, vol. 1 of Advanced Series on Complex Systems, World Scientific, Singapore, 1986.

[32] L. Zhao and E. E. N. Macau, "A network of dynamically coupled chaotic maps for scene segmentation," IEEE Transactions on Neural Networks, vol. 12, no. 6, pp. 1375-1385, 2001.

[33] Y. Kuramoto, Chemical Oscillations, Waves, and Turbulence, vol. 19 of Springer Series in Synergetics, Springer, Berlin, Germany, 1984. 


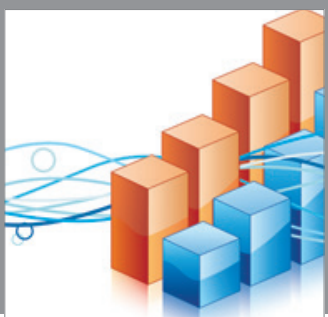

Advances in

Operations Research

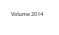

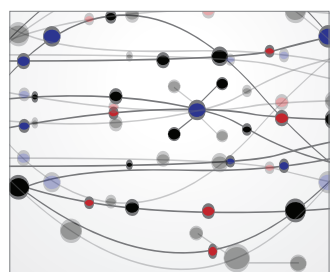

\section{The Scientific} World Journal
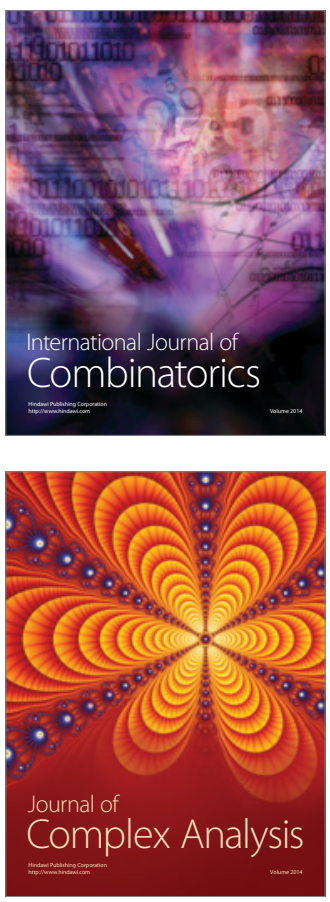

International Journal of

Mathematics and

Mathematical

Sciences
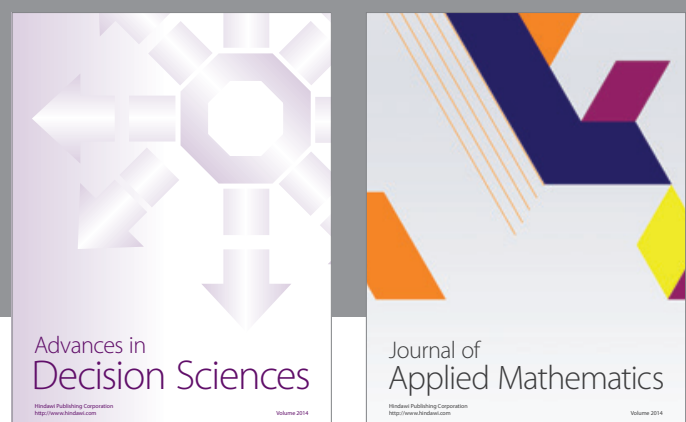

Journal of

Applied Mathematics
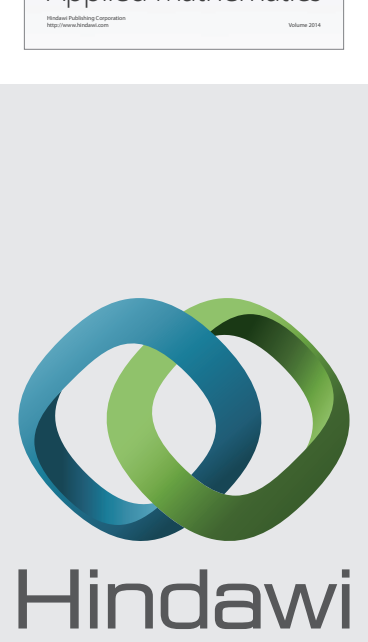

Submit your manuscripts at http://www.hindawi.com
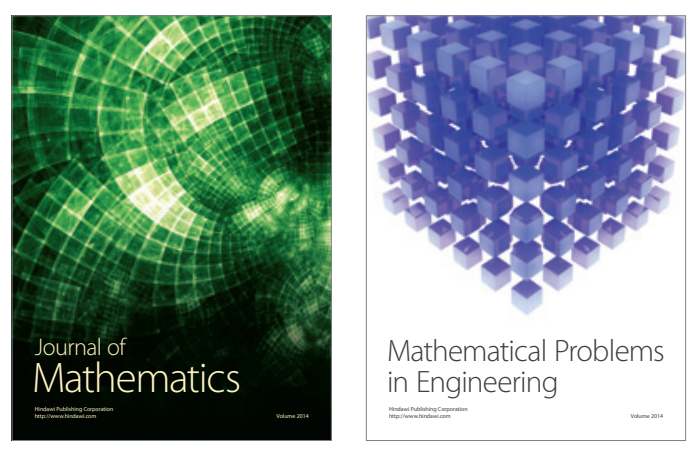

Mathematical Problems in Engineering
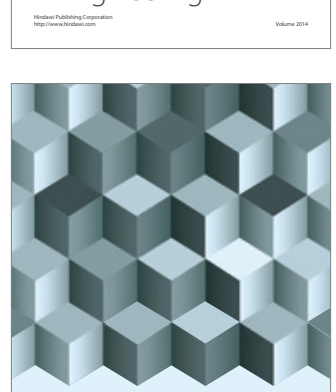

Journal of

Function Spaces
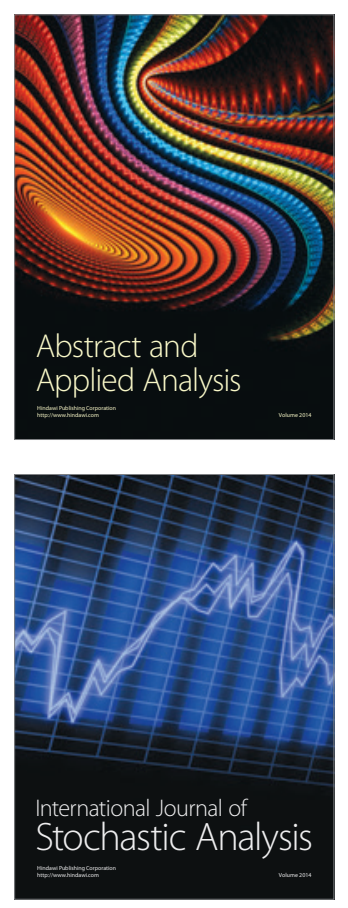

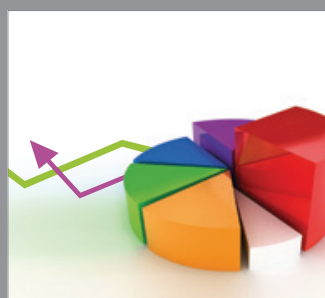

ournal of

Probability and Statistics

Promensencen
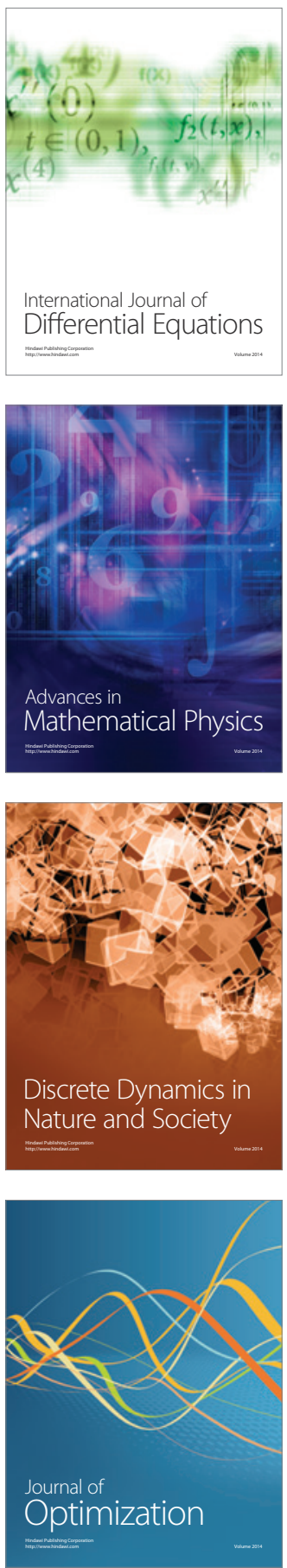